\title{
Reducing discretionary food and beverage intake in early childhood: a systematic review within an ecological framework
}

\author{
Brittany J Johnson ${ }^{1, *}$, Gilly A Hendrie ${ }^{2}$ and Rebecca K Golley ${ }^{1}$ \\ 'Division of Health Sciences, Sansom Institute for Health Research, University of South Australia, North Terrace, GPO \\ Box 2471, Adelaide, SA 5001, Australia: ${ }^{2}$ Commonwealth Scientific Industrial Research Organisation Food and \\ Nutrition Flagship, Adelaide, South Australia, Australia
}

Submitted 10 April 2015: Final revision received 14 September 2015: Accepted 18 September 2015: First published online 21 0ctober 2015

\begin{abstract}
Objective: To systematically review the literature and map published studies on 4-8-year-olds' intake of discretionary choices against an ecological framework (ANalysis Grid for Environments Linked to Obesity; ANGELO).

Design: Articles were identified through database searches (PubMed, PyscINFO ${ }^{\circledR}$, Web of Science) in February and March 2014 and hand-searching reference lists. Studies were assessed for methodological quality and mapped against the ANGELO framework by environment size (macro and micro setting) and type (physical, economic, policy and socio-cultural influences).

Setting: Studies were conducted in the USA ( $n$ 18), Australia ( $n$ 6), the UK ( $n$ 3), the Netherlands ( $n$ 3), Belgium ( $n$ 1), Germany $(n 1)$ and Turkey $(n 1)$.

Subjects: Children aged 4-8 years, or parents/other caregivers.

Results: Thirty-three studies met the review criteria (observational $n 23$, interventions $n$ 10). Home was the most frequently studied setting (67\% of exposures/strategies), with the majority of these studies targeting family policy-type influences (e.g. child feeding practices, television regulation). Few studies were undertaken in government $(5.5 \%)$ or community (11\%) settings, or examined economic-type influences ( $0 \%)$. Of the intervention studies only four were categorised as effective.

Conclusions: The present review is novel in its focus on mapping observational and intervention studies across a range of settings. It highlights the urgent need for high-quality research to inform interventions that directly tackle the factors influencing children's excess intake of discretionary choices. Interventions that assist in optimising a range of environmental influences will enhance the impact of future public health interventions to improve child diet quality.
\end{abstract}

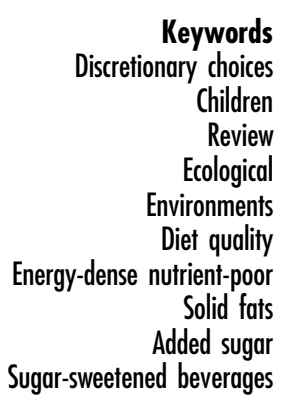

Consumers globally are exposed to an abundance of energy-dense, nutrient-poor foods and beverages, which can be referred to as 'discretionary foods' or 'discretionary choices $^{(1-5)}$. Examples of such foods are sugar-sweetened beverages, processed meats, fried foods, pastries and cakes. The Australian Dietary Guidelines recommend limiting the intake of (discretionary) foods containing saturated fat, added sugar, salt and alcohol that are not required as part of a dietary pattern that promotes health and well-being ${ }^{(5)}$.

Overconsumption of discretionary choices is associated with increased risk of obesity, CVD, type 2 diabetes, some cancers and dental caries ${ }^{(5)}$. The risk of these chronic conditions and related dietary patterns begin in childhood and track into adult life ${ }^{(5,6)}$. This is of particular concern given the sizeable chronic disease burden and high rates of obesity across the lifespan ${ }^{(7)}$. Overconsumption of discretionary choices can also displace core foods (fruit, vegetables, dairy, lean meats, whole grains) from the diet, further increasing the risk of nutrient deficiencies, obesity and chronic disease ${ }^{(8,9)}$.

National intake data across the Western world show that the majority of children are not meeting the recommended intakes of core foods and are overconsuming discretionary choices $^{(10-13)}$. According to US data, $99 \cdot 9 \%$ of 4-8-yearold children are consuming above the maximum recommended energy allowance from discretionary choices ${ }^{(10)}$. This statistic aligns with Australian data, which show that 
consumption of discretionary choices currently contributes about $36 \%$ of children's energy intake, with intakes increasing with age from $30.2 \%$ in 2-3-year-olds to $37.5 \%$ in 4-8-year-olds ${ }^{(11,12)}$. Early childhood is an opportune time to intervene to moderate intake of discretionary choices and potentially increase core foods to establish healthier dietary patterns.

Individual and environmental factors influence food provision to, and the dietary intake of, young children

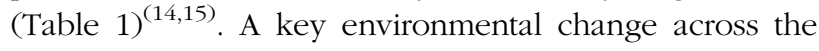
ages of 4-8 years is the transition from home or child care to formal education settings (pre-school, junior primary school) ${ }^{(16)}$. While starting school widens children's environmental influences ${ }^{(15,16)}$, children still rely predominantly on their caregivers for food provision and the home setting remains an important environment influencing food intake. Creating supportive eating environments to minimise children's intake of discretionary choices, in all settings where children spend their time, will enhance the impact and sustainability of nutrition promotion and obesity prevention efforts.

Discretionary choices have received little direct attention in the paediatric nutrition literature. To date, the literature has instead focused on general healthy eating interventions for obesity prevention and management ${ }^{(17-19)}$ or nutrition promotion interventions targeting fruit and vegetable intake ${ }^{(20)}$. Children's exposure to food marketing is one area where there is some evidence of an association with discretionary choices intake ${ }^{(21)}$; however, more research is needed to understand the factors that influence children's intakes of these nutritionally poor foods and identify other targets for intervention.

The present review aims to identify and map the range of environmental predictors that influence children's intake of discretionary choices. The review focuses on observational and intervention studies targeting children aged $4-8$ years or their caregivers, with a clear emphasis on discretionary choices, measuring children's intakes of these foods directly or via proxy. The review objectives are:

1. To identify and map the current literature on predictors of 4-8-year-old children's intake of discretionary choices using an ecological framework.

2. To provide recommendations for the 'next steps' of future research by highlighting gaps in the current body of evidence.

\section{Methods}

A systematic review process ${ }^{(22)}$ was implemented to identify original published studies meeting the inclusion criteria listed below. Studies were then critiqued and synthesised to answer the review objectives. No publication date limit was set to access all literature published in English meeting the review inclusion criteria.

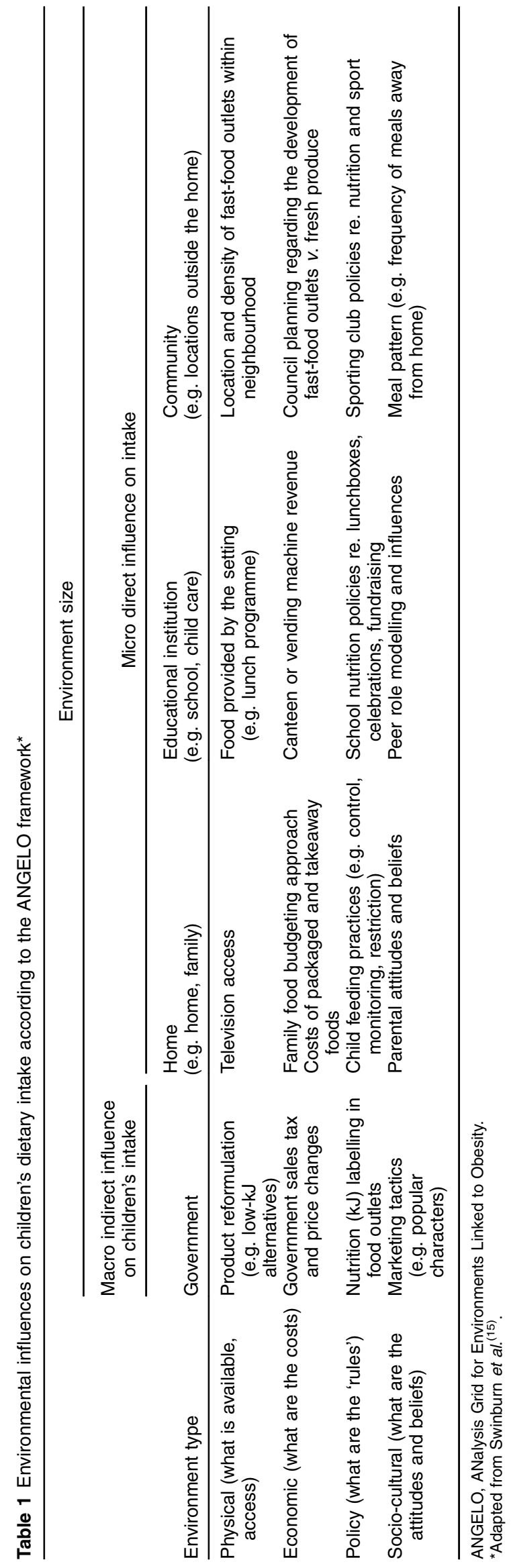




\section{Inclusion and exclusion criteria}

\section{Population}

The population under review was healthy children, with an age range or mean age between 4.0 and 7.9 years, or their parents/other caregivers (e.g. grandparents, educators). Clinical samples were excluded (e.g. anaemia, cystic fibrosis, diabetes).

\section{Predictors (intervention or comparator)}

All correlates modifiable in a nutrition promotion context were included (e.g. child feeding practices, hours of television use). Descriptive studies or studies focusing on non-modifiable correlates (e.g. age, ethnicity) were excluded. Studies that focused on overweight/obesity management were excluded as the focus of the review was on broader public health diet quality and obesity prevention.

\section{Outcome measures}

Primary outcomes were children's intakes (or proxy) of discretionary foods or beverages, or related components (i.e. energy, saturated fat, sugar or sodium). Proxy measures included availability (e.g. food access, parent intake), provision (e.g. parenting control), preferences (e.g. child requests), behaviour (e.g. meal pattern, child feeding practices) and purchasing (e.g. receipts).

Secondary outcomes included knowledge/attitudes/ beliefs of the child or carer. Studies with a secondary outcome were included only if they also contained at least one primary outcome measure.

\section{Study design and type}

To capture the breadth of work on discretionary choices, observational and intervention studies conducted in any of the settings outlined in Table 1 were included. Non-human (e.g. animal model, laboratory), methodological (e.g. food record tool, protocol only) and non-primary studies (e.g. review papers, commentary) were excluded.

\section{Search strategy and selection process}

Search terms were combined under the following headings:

1. Population (e.g. child* or preschool), and

2. Intervention (e.g. 'nutrition education' or 'behaviour change' or 'dietary intake' or 'eating pattern*'), and

3. Outcomes (e.g. 'energy-dense' or 'non-core food' or 'high calories' or 'sugar-sweetened beverages' or 'fast food' or 'saturated fat' or 'added sugar' or 'salt' or 'energy intake').

4. Limits applied: English.

Terms were trialled in PubMed. PubMed, PsycINFO ${ }^{\circledR}$ and Web of Science were systematically searched in February (PubMed, PsycINFO ${ }^{\circledR}$ ) and March (Web of Science) 2014, and citations identified from other sources (Fig. 1). Title, abstract and full-text screening was completed by two reviewers; in cases of uncertainty, a third reviewer was consulted and consensus reached.

\section{Data extraction}

Data extracted included: study details (identification, site, setting, study design, study duration, participants), discretionary choices targeted, exposure/intervention strategies, environmental influences, outcome assessment method, key results and quality assessment tool score. To ensure consistency of data extracted, $10 \%$ of studies were rechecked by the single reviewer. Due to the heterogeneity in the design, exposures/strategies and outcomes of included studies, a meta-analysis was not undertaken. Statistical significance (of $P<0.05$ ) was used to guide the review narrative. Results are presented in a narrative summary, with overall direction and confidence of the association of the study findings summarised using the following symbols: significant negative association, - - ; non-significant negative association, -; null association, 0; non-significant positive association, +; significant positive association, ++; inconsistent associations, $\sim$ (Tables 3 and 4). Non-significant findings were highlighted to examine whether consistent effect sizes (direction) were present across studies and to consider where an increase in sample size or better study design may have achieved statistical significance.

\section{Study quality}

A single reviewer assessed study quality using the standardised Effective Public Health Practice Project (EPHPP) Quality Assessment Tool ${ }^{(23)}$. The tool scores study quality (strong, moderate, weak) across six areas: selection bias, study design, confounders, blinding, data collection methods, withdrawals and drop-outs ${ }^{(23)}$. An overall quality rating is assigned: strong (four strong ratings and no weak ratings), moderate (less than four strong ratings and one weak rating) or weak (two or more weak ratings) ${ }^{(23)}$. In an assessment of 213 quality assessment tools, this tool was identified as useful for systematic reviews that evaluate randomised and non-randomised intervention studies ${ }^{(24)}$. To enable comparison of study quality across study design, the tool was adapted to allow for use with observational studies, as well as interventions. Adaptations to the tool included description of covariates rather than difference between groups prior to interventions, and adjustments for cross-sectional design with no drop-outs and one data collection time point.

\section{Mapping of environmental influence}

Observational study correlates were termed 'exposures' and intervention strategies were termed 'strategies'. Study exposures/strategies were mapped using the ANalysis Grid for Environments Linked to Obesity (ANGELO), an ecological framework that identifies a range of environment sizes (i.e. macro and micro setting) and environment types (e.g. physical, economic, policy, socio-cultural) that likely influence 


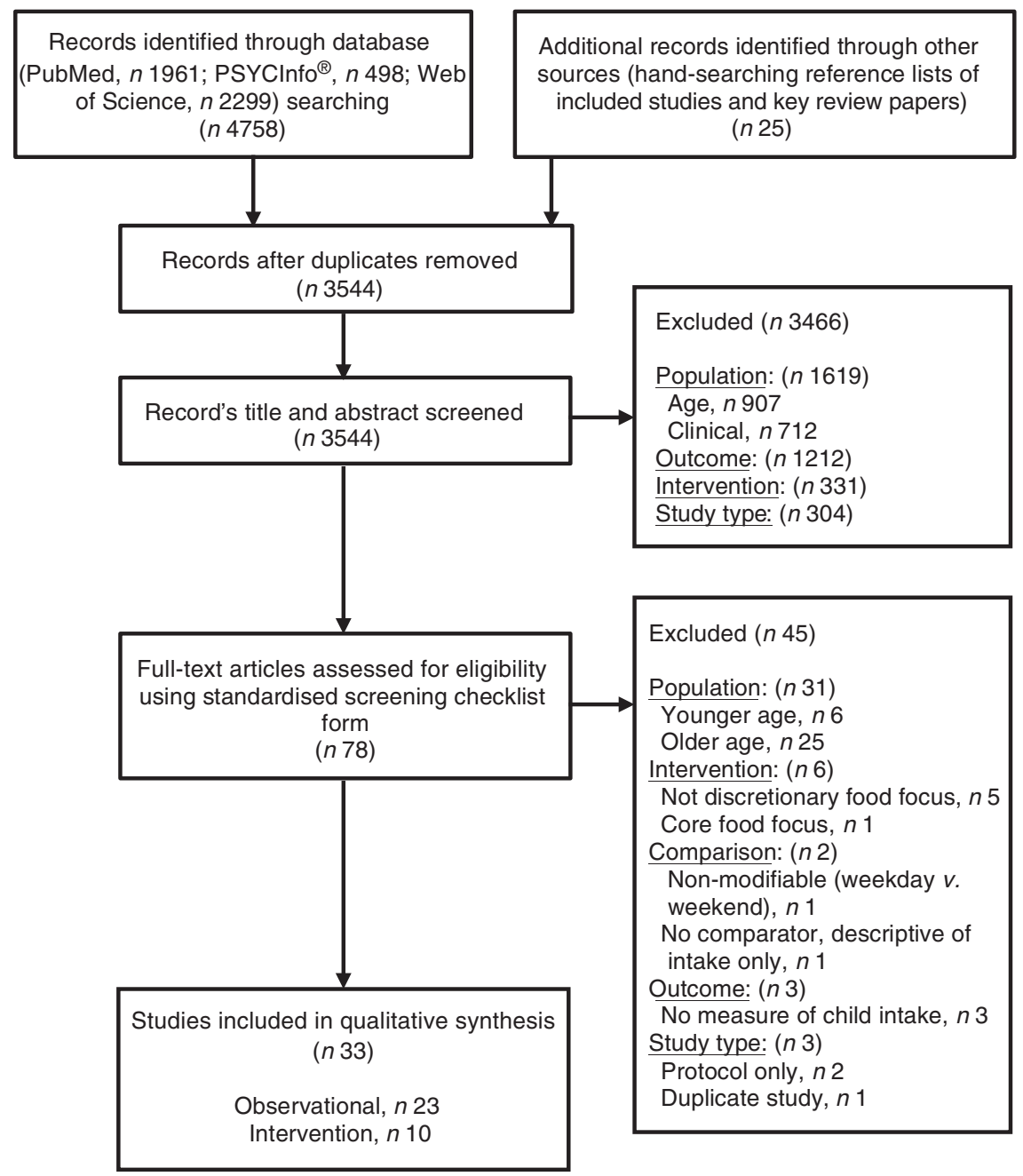

Fig. 1 PRISMA Statement flow diagram indicating number of studies included at each phase of the review (PRISMA, Preferred Reporting Items for Systematic Reviews and Meta-Analyses)

children's discretionary choices intake, and has been described elsewhere in detail ${ }^{(15)}$. A coding system was developed in order to systematically assign a type of exposure/strategy to one of the ANGELO framework environments. A single reviewer hand-coded exposures/strategies using Table 1; any uncertainties by the reviewer were discussed with all reviewers. Where one study included multiple exposures/ strategies all relevant codes were applied. To simplify the coding process using the ANGELO framework only the primary environment type and size of the exposure/strategy were coded. For example, frequency of meals consumed away from home (termed 'meal pattern') was coded as a socio-cultural influence within the community. Another example is kilojoule labelling of food outlet menus, which was coded as a policy influence within the government setting.

\section{Results}

Thirty-three studies met the review criteria (Fig. 1). Over half of studies were based in the USA $(n 18)^{(2,3,25-40)}$ or Australia $(n 6)^{(41-46)}$. Twenty-three were cross-sectional, observational studies $^{(1,2,25-35,41-43,47-53)}$ (see online supplementary material, Supplementary Table 1), with an average sample size of 563 (range $41^{(31)}-2074^{(50)}$ ). There were ten interventions (randomised controlled trials, $n 8^{(3,36,38,39,44-46,54)}$; case-control trial, $n 1^{(37)}$; cohort study, $n 1^{(40)}$; see online supplementary material, Supplementary Table 2), with an average sample size of 210 participants (range $29^{(38)}-560^{(46)}$ ). No studies rated high in methodological quality. Several potential sources of bias including participant selection, study design and data collection methods were common across study design. However, intervention studies were more likely to rate highly (60\% moderate, $40 \%$ weak) compared with observational studies ( $4 \%$ moderate, $96 \%$ weak).

\section{Ecological mapping of studies}

For each study, the exposure variables (in observational studies) or strategies (in intervention studies) were coded and mapped against the ANGELO framework (Table 2). In total fifty-five exposures/strategies were mapped, which included 
Table 2 Spread of current literature (observational and intervention studies) on 4-8-year-olds' intake of discretionary choices by ANGELO framework (environment size and type)*

\begin{tabular}{|c|c|c|c|c|c|}
\hline \multirow[b]{3}{*}{ Environment type } & \multicolumn{5}{|c|}{ Environment size } \\
\hline & \multirow{2}{*}{$\frac{\text { Macro }}{\text { Government }}$} & \multicolumn{3}{|c|}{ Micro } & \multirow[b]{2}{*}{ Total } \\
\hline & & Home & Educational institution & Community & \\
\hline \multicolumn{6}{|c|}{ Observational ( $71 \%$ of exposures measured) } \\
\hline Physical & - & $10 \dagger$ & 1 & 1 & 12 \\
\hline Economic & - & - & - & - & - \\
\hline Policy & - & 16 & 1 & - & 17 \\
\hline Socio-cultural & - & 5 & - & 5 & 10 \\
\hline Total & - & 31 & 2 & 6 & 39 \\
\hline \multicolumn{6}{|c|}{ Intervention ( $29 \%$ of strategies evaluated) } \\
\hline Physical & 1 & 3 & 2 & - & 6 \\
\hline Economic & - & - & - & - & - \\
\hline Policy & 2 & 2 & 2 & - & 6 \\
\hline Socio-cultural & - & 1 & 3 & - & 4 \\
\hline Total & 3 & 6 & 7 & - & 16 \\
\hline
\end{tabular}

ANGELO, ANalysis Grid for Environments Linked to Obesity Framework.

*Studies may investigate multiple exposures or strategies; therefore one study may represent multiple environments.

†Number of studies including the exposure or strategy.

thirty-nine exposures from twenty-three observational studies and sixteen strategies from ten intervention studies.

Studies were mapped against environmental size (i.e. macro and micro setting). There were few studies within the government setting, with only three intervention strategies ${ }^{(36-38)}$ and no observational exposures evaluated in this setting. Most studies were undertaken within the home setting (thirty-seven exposures/strategies out of fifty-five), primarily in observation studies (thirty-one out of thirty-seven). The majority of research within educational institutions has been from intervention studies; seven strategies ${ }^{(3,39,46,54)}$ were identified compared with two exposures from observational studies ${ }^{(53)}$. A total of six exposures have been examined within the community setting, all from observational research ${ }^{(26,27,29,41,48,51)}$.

Studies were also mapped by environment type (i.e. physical, economic, policy, socio-cultural) by study design. Within observational studies, policy-type exposures were most common (seventeen out of thirty-nine), followed by physical (twelve out of thirty-nine) and socio-cultural (ten out of thirty-nine). No economic-type exposures were included in any observational studies. Within interventions, physical (six out of sixteen) and policy-type (six out of sixteen) strategies were most common. Again, economic strategies were not included in any interventions targeting children's discretionary choices intake.

\section{Ecological view: summary of the relationships in observational studies}

Table 3 summarises the results of observational studies by environmental size and type. Six observational studies were conducted within the community setting, five focused on discretionary choices as a whole and one on sugar-sweetened beverages only. Five observational studies focused on meal patterns (socio-cultural influence), of which three reported a significant positive relationship with discretionary choices intake ${ }^{(27,29,41)}$. That is, higher frequency of meals or snacks consumed out of the home (e.g. fast food, restaurant, home of friends/relatives) was significantly associated with higher intake of discretionary choices ${ }^{(27,29,41)}$. One community-based observational study focused on food availability (physical influence) and reported no relationship between availability of discretionary choices within the community and children's intake $^{(48)}$.

There was one observational study within the educational setting, which reported that greater food availability (physical influence) was significantly associated with higher intake of discretionary choices ${ }^{(53)}$. The study also reported that the absence of a nutrition policy was associated with higher intake of discretionary choices, although the result did not reach statistical significance ${ }^{(53)}$.

Nineteen observational studies measured exposures within the home setting, fourteen of which focused on discretionary foods as a whole, with two targeting snacks, two with a nutrient focus and one focusing only on sugar-sweetened beverages. Within the home setting, sixteen studies examined policy-type exposures, primarily relating to child feeding practices. Four studies found that higher level of parental control (pressure) was related to higher children's intake of discretionary choices ${ }^{(28,30,42,47)}$. In all four of these studies, more than three discretionary choices were measured. Some studies also noted that higher parental monitoring, a desired feeding practice, was associated with lower intake of discretionary choices $^{(2,27,28,30,47,52)}$. Other policy-type studies in the home focused on television regulation targeting various discretionary choices ${ }^{(1,25,27,31,32,34)}$. Of these studies, four out of six found higher discretionary choices intake 
Table 3 Summary of observational studies targeting 4-8-year-olds' intake of discretionary choices

\begin{tabular}{|c|c|c|c|}
\hline Reference, country, study design, quality* & ANGELO framework (environment size and type) & $\begin{array}{l}\text { Discretionary } \\
\text { choices targeted }\end{array}$ & $\begin{array}{l}\text { Results } \\
\text { summary† }\end{array}$ \\
\hline $\begin{array}{l}\text { Micro: Community } \\
\text { Ayala et al. }(2008)^{(29)} \\
\text { USA } \\
\text { Cross-sectional } \\
\text { Weak }\end{array}$ & $\begin{array}{l}\text { Socio-cultural: meal pattern (higher frequency of meals } \\
\text { away from home) }\end{array}$ & $\mathrm{DC} \S$ & ++ \\
\hline $\begin{array}{l}\text { Buck et al. }(2013)^{(48)} \\
\text { Germany } \\
\text { Cross-sectional } \\
\text { Weak }\end{array}$ & $\begin{array}{l}\text { Physical: food availability } \\
\text { (higher DC availability within community) }\end{array}$ & DC & 0 \\
\hline $\begin{array}{l}\text { Erinsho et al. }(2013)^{(26)} \ddagger \\
\text { USA } \\
\text { Cross-sectional } \\
\text { Weak }\end{array}$ & Socio-cultural: meal pattern & DC & + \\
\hline $\begin{array}{l}\text { Kerr et al. }(2010)^{(51)} \\
\quad \text { Ireland } \\
\text { Cross-sectional } \\
\text { Weak }\end{array}$ & Socio-cultural: meal pattern & DC & $\sim$ \\
\hline $\begin{array}{l}\text { Lopez et al. }(2012)^{(27)} \ddagger \\
\text { USA } \\
\text { Cross-sectional } \\
\text { Weak }\end{array}$ & Socio-cultural: meal pattern & SSB\| & ++ \\
\hline $\begin{array}{l}\text { Spurrier et al. }(2008)^{(41)} \ddagger \\
\quad \text { Australia } \\
\text { Cross-sectional } \\
\text { Weak }\end{array}$ & Socio-cultural: meal pattern & DC & ++ \\
\hline $\begin{array}{l}\text { Micro: Educational institution } \\
\text { Vereecken et al. }(2008)^{(53)} \\
\text { Belgium } \\
\text { Cross-sectional } \\
\text { Weak }\end{array}$ & $\begin{array}{l}\text { Physical: food availability } \\
\text { Policy: nutrition policy }\end{array}$ & DC & + \\
\hline $\begin{array}{l}\text { Micro: Home } \\
\text { Aguirre et al. }(2012)^{(28)} \\
\text { USA } \\
\text { Cross-sectional } \\
\text { Weak }\end{array}$ & $\begin{array}{l}\text { Policy: child feeding practices (higher control and } \\
\text { restriction and lower monitoring) }\end{array}$ & $\mathrm{DC}$ & ++ \\
\hline $\begin{array}{l}\text { Aktas Arnas }(2006)^{(1)} \\
\text { Turkey } \\
\text { Cross-sectional } \\
\text { Weak }\end{array}$ & $\begin{array}{l}\text { Physical: TV access (higher child TV viewing) } \\
\text { Policy: TV regulation (higher parental } \\
\text { regulation of child TV viewing) }\end{array}$ & DC & 0 \\
\hline $\begin{array}{l}\text { Andaya et al. }(2011)^{(25)} \\
\text { USA } \\
\text { Cross-sectional } \\
\text { Weak }\end{array}$ & $\begin{array}{l}\text { Physical: TV access } \\
\text { Policy: meal pattern, TV regulation } \\
\text { Socio-cultural: mealtime structure } \\
\text { (higher frequency of snacking) }\end{array}$ & $\begin{array}{l}\text { Soda/soft drinks, } \\
\text { savoury snacks }\end{array}$ & $\sim$ \\
\hline $\begin{array}{l}\text { Arredondo et al. }(2006)^{(30)} \\
\text { USA } \\
\text { Cross-sectional } \\
\text { Weak }\end{array}$ & Policy: child feeding practices & $\mathrm{DC}$ & $\sim$ \\
\hline $\begin{array}{l}\text { Brown et al. }(2008)^{(47)} \\
\text { UK } \\
\text { Cross-sectional } \\
\text { Weak }\end{array}$ & Policy: child feeding practices & $\mathrm{DC}$ & $\sim$ \\
\hline $\begin{array}{l}\text { Campbell et al. (2006) } \\
\quad \text { Australia } \\
\text { Cross-sectional } \\
\text { Weak }\end{array}$ & $\begin{array}{l}\text { Physical: food availability, TV access } \\
\text { Policy: child feeding practices } \\
\text { Socio-cultural: mealtime structure }\end{array}$ & DC & ++ \\
\hline $\begin{array}{l}\text { Campbell et al. }(2010)^{(43)} \\
\text { Australia } \\
\text { Cross-sectional } \\
\text { Moderate }\end{array}$ & $\begin{array}{l}\text { Socio-cultural: parental beliefs/attitudes } \\
\text { (higher positive DC-related beliefs) }\end{array}$ & DC & 0 \\
\hline $\begin{array}{l}\text { Erinsho et al. }(2013)^{(26)} \ddagger \\
\text { USA } \\
\text { Cross-sectional } \\
\text { Weak }\end{array}$ & Physical: food availability, TV access & $\mathrm{DC}$ & + \\
\hline $\begin{array}{l}\text { Galst and White }(1976)^{(31)} \\
\text { USA } \\
\text { Cross-sectional } \\
\text { Weak }\end{array}$ & $\begin{array}{l}\text { Physical: TV access } \\
\text { Policy: TV regulation, parenting } \\
\text { (higher positive parenting practices) }\end{array}$ & $\mathrm{DC}$ & ++ \\
\hline
\end{tabular}




\begin{tabular}{|c|c|c|c|}
\hline Reference, country, study design, quality* & ANGELO framework (environment size and type) & $\begin{array}{l}\text { Discretionary } \\
\text { choices targeted }\end{array}$ & $\begin{array}{l}\text { Results } \\
\text { summary† }\end{array}$ \\
\hline $\begin{array}{l}\text { Gubbels et al. }(2011)^{(50)} \\
\text { Netherlands } \\
\text { Cross-sectional } \\
\text { Weak }\end{array}$ & Policy: meal pattern & DC & 0 \\
\hline $\begin{array}{l}\text { Gubbels et al. }(2012)^{(49)} \\
\text { Netherlands } \\
\text { Cross-sectional } \\
\text { Weak }\end{array}$ & Policy: child feeding practices & Energy, sugar & + \\
\hline $\begin{array}{l}\text { Isler et al. }(1987)^{(32)} \\
\text { USA } \\
\text { Cross-sectional } \\
\text { Weak }\end{array}$ & $\begin{array}{l}\text { Physical: TV access } \\
\text { Policy: TV regulation, parenting }\end{array}$ & $\mathrm{DC}$ & ++ \\
\hline $\begin{array}{l}\text { Lopez et al. }(2012)^{(27)} \ddagger \\
\text { USA } \\
\text { Cross-sectional } \\
\text { Weak }\end{array}$ & $\begin{array}{l}\text { Physical: TV access } \\
\text { Policy: child feeding practices, TV regulation }\end{array}$ & SSB & $\sim$ \\
\hline $\begin{array}{l}\text { Murashima et al. }(2012)^{(2)} \\
\text { USA } \\
\text { Cross-sectional } \\
\text { Weak }\end{array}$ & Policy: child feeding practices & DC & $\sim$ \\
\hline $\begin{array}{l}\text { Ogden et al. }(2006)^{(52)} \\
\text { UK } \\
\text { Cross-sectional } \\
\text { Weak }\end{array}$ & Policy: child feeding practices & $\mathrm{DC}$ & $\sim$ \\
\hline $\begin{array}{l}\text { Spurrier et al. }(2008)^{(41)} \ddagger \\
\quad \text { Australia } \\
\text { Cross-sectional } \\
\text { Weak }\end{array}$ & $\begin{array}{l}\text { Physical: food availability, TV access } \\
\text { Policy: child feeding practices } \\
\text { Socio-cultural: mealtime structure }\end{array}$ & $\mathrm{DC}$ & $\sim$ \\
\hline $\begin{array}{l}\text { Tabak }(2011)^{(33)} \\
\text { USA } \\
\text { Cross-sectional } \\
\text { Weak }\end{array}$ & $\begin{array}{l}\text { Policy: child feeding practices } \\
\text { Socio-cultural: mealtime structure }\end{array}$ & $\mathrm{DC}$ & $\sim$ \\
\hline $\begin{array}{l}\text { Taras et al. }(1989)^{(34)} \\
\text { USA } \\
\text { Cross-sectional } \\
\text { Weak }\end{array}$ & $\begin{array}{l}\text { Physical: TV access } \\
\text { Policy: TV regulation }\end{array}$ & $\begin{array}{l}\text { Energy, saturated fat, } \\
\text { sugar, salt }\end{array}$ & $\sim$ \\
\hline $\begin{array}{l}\text { Wroten et al. }(2012)^{(35)} \\
\text { USA } \\
\text { Cross-sectional } \\
\text { Weak }\end{array}$ & Physical: food availability & $\begin{array}{l}\text { Sweet }^{\star \star} \text { and savoury } \\
\text { snacks }\end{array}$ & ++ \\
\hline
\end{tabular}

ANGELO, ANalysis Grid for Environments Linked to Obesity; DC, discretionary choices; TV, television; SSB, sugar-sweetened beverages.

${ }^{*}$ Study methodology quality is assessed using the Effective Public Health Practice Project Quality Assessment Tool, with an overall quality rating assigned as: strong (four strong ratings and no weak ratings), moderate (less than four strong ratings and one weak rating) or weak (two or more weak ratings) ${ }^{(23)}$.

†Direction and confidence of the association are summarised using the following symbols: significant negative association, $--;$ non-significant negative association, - null association, 0; non-significant positive association, +; significant positive association, ++; inconsistent association, $\sim$.

¥The study covered multiple environmental sizes.

§Includes more than three discretionary food choices.

IIIncludes soft drinks, energy drinks, fruit juice and other flavoured drinks.

IIncludes salty and high-fat snacks, potato chips and other savoury/packaged snacks.

${ }^{\star \star}$ Includes confectionery, cakes, biscuits and other sweet snacks.

was significantly associated with higher television use $^{(27,31,32,34)}$. Television access (physical influence) was also observed in the home in nine studies, with six targeting a range of discretionary choices. Higher access to television within the home was associated with significantly higher intake of discretionary choices in five of the studies ${ }^{(27,31,32,34,42)}$.

\section{Ecological view: summary of association from intervention studies}

Intervention studies were also categorised by environmental size and type (Table 4). Three interventions were conducted within the government setting; two focused on nutrition labelling, specifically fast-food menu labelling (policy influence), and targeted single discretionary food items (e.g. fast food) rather than a variety of discretionary choices $^{(36-38)}$. One study reported a significant, negative relationship between the policy strategy and intake; that is, the introduction of the policy was associated with a significant reduction in children's fast-food intake ${ }^{(36)}$. One intervention focused on food reformulation (physical influence) and reported a negative, but non-significant, association with children's snack food intake ${ }^{(38)}$.

Four interventions were set within educational institutions, and three targeted socio-cultural influences 
Table 4 Summary of intervention studies targeting 4-8-year-olds' intake of discretionary choices

\begin{tabular}{|c|c|c|c|}
\hline Reference, country, study design, quality* & ANGELO framework (environment size and type) & $\begin{array}{l}\text { Discretionary } \\
\text { choices targeted }\end{array}$ & $\begin{array}{l}\text { Results } \\
\text { summary } †\end{array}$ \\
\hline $\begin{array}{l}\text { Macro: Government } \\
\text { Birch et al. }(1993)^{(38)} \\
\text { USA } \\
\text { RCT } \\
\text { Weak }\end{array}$ & $\begin{array}{l}\text { Physical: food reformulation (reduction in DC-associated } \\
\text { nutrients) }\end{array}$ & $\begin{array}{l}\text { Sweetł and savoury } \\
\text { snacks§ }\end{array}$ & - \\
\hline $\begin{array}{l}\text { Stutts et al. }(2011)^{(37)} \\
\text { USA } \\
\text { RCT } \\
\text { Weak }\end{array}$ & $\begin{array}{l}\text { Policy: nutrition labelling (addition of DC-associated } \\
\text { nutrient labelling) }\end{array}$ & Fast food & - \\
\hline $\begin{array}{l}\text { Tandon et al. }(2010)^{(36)} \\
\text { USA } \\
\text { RCT } \\
\text { Moderate }\end{array}$ & Policy: nutrition labelling & Fast food & -- \\
\hline Micro: Educational institution & & & \\
\hline $\begin{array}{l}\text { Acran et al. }(2013)^{(39)} \\
\text { USA } \\
\text { RCT } \\
\text { Moderate }\end{array}$ & $\begin{array}{l}\text { Policy: teacher's classroom food-related practices } \\
\text { (reduction in behaviours using DC) } \\
\text { Socio-cultural: teacher beliefs (increase in positive } \\
\text { DC-related beliefs) }\end{array}$ & $\begin{array}{l}\text { Candy, fast food, } \\
\text { SSBII }\end{array}$ & -- \\
\hline $\begin{array}{l}\text { Bevelander et al. }(2012)^{(54)} \\
\text { Netherlands } \\
\text { RCT } \\
\text { Moderate }\end{array}$ & Socio-cultural: peer influence (positive peer modelling) & Sweet snacks & 0 \\
\hline $\begin{array}{l}\text { Musher-Eizenman et al. }(2010)^{(3)} \\
\text { USA } \\
\text { RCT } \\
\text { Moderate }\end{array}$ & Physical: food availability & Snack foods & - \\
\hline $\begin{array}{l}\text { Zask et al. }(2012)^{(46)} \\
\text { Australia } \\
\text { RCT } \\
\text { Weak }\end{array}$ & $\begin{array}{l}\text { Physical: food availability } \\
\text { Policy: nutrition policy } \\
\text { Socio-cultural: parental knowledge/attitudes/beliefs }\end{array}$ & DCI & -- \\
\hline $\begin{array}{l}\text { Micro: Home } \\
\text { Dickin et al. }(2014)^{(40)} \\
\text { USA } \\
\text { Cohort } \\
\text { Weak }\end{array}$ & $\begin{array}{l}\text { Physical: food availability/SSB substitution water, } \\
\text { low-fat milk }\end{array}$ & SSB, fast food & -- \\
\hline $\begin{array}{l}\text { Duncanson et al. }(2013)^{(44)} \\
\text { Australia } \\
\text { RCT } \\
\text { Moderate }\end{array}$ & $\begin{array}{l}\text { Physical: food availability } \\
\text { Policy: child feeding practices }\end{array}$ & DC & - \\
\hline $\begin{array}{l}\text { Fletcher et al. }(2013)^{(45)} \\
\text { Australia } \\
\text { RCT } \\
\text { Moderate }\end{array}$ & $\begin{array}{l}\text { Physical: food availability } \\
\text { Policy: child feeding practices } \\
\text { Socio-cultural: mealtime structure }\end{array}$ & DC & - \\
\hline
\end{tabular}

ANGELO, ANalysis Grid for Environments Linked to Obesity; RCT, randomised controlled trial; SSB, sugar-sweetened beverages; DC, discretionary choices. *Study methodology quality is assessed using the Effective Public Health Practice Project Quality Assessment Tool, with an overall quality rating assigned as: strong (four strong ratings and no weak ratings), moderate (less than four strong ratings and one weak rating) or weak (two or more weak ratings) (23).

†Direction and confidence of the association are summarised using the following symbols: significant negative association, $--;$ non-significant negative association, - null association, 0; non-significant positive association, +; significant positive association, ++; inconsistent association, $~$.

†Includes confectionery, cakes, biscuits and other sweet snacks.

§Includes salty and high-fat snacks, potato chips and other savoury/packaged snacks.

IIIncludes soft drinks, energy drinks, fruit juice and other flavoured drinks.

IIncludes more than three discretionary food choices.

(e.g. teacher or parent beliefs and attitudes, influence of peers), two examined policy strategies (e.g. nutrition policy) and two included physical (e.g. food availability) strategies ${ }^{(3,39,46,54)}$. These strategies tended to focus on a smaller number of discretionary choices, such as snack foods, sweet snacks or confectionery ${ }^{(3,39,54)}$. Two (of four) studies within educational institutions reported a significant reduction in discretionary choices intake, both included policy and socio-cultural strategies ${ }^{(39,46)}$.
Three studies were conducted within the home, all included elements of addressing the physical influence of discretionary choices availability ${ }^{(40,44,45)}$. All reported favourable results towards a reduction in children's intake of these foods ${ }^{(40,44,45)}$; however, only one study reported a significant reduction in discretionary choices ${ }^{(40)}$. This latter study focused on food substitution but was limited to specific discretionary foods, for example replacing sugarsweetened beverages by water or low-fat milk ${ }^{(40)}$. 


\section{Discussion}

The present review evaluated the observational and intervention literature to map the current evidence targeting a reduction in children's intake of discretionary choices. This aim was focused on children aged 4-8 years due to the expanding environmental influences present during the transition to formal education ${ }^{(16)}$.

The review identified only thirty-three studies across the wide range of settings which could potentially influence children's discretionary choices intake. This was surprising given the health and diet quality concerns regarding discretionary choices ${ }^{(5)}$. When compared with the breadth of literature regarding improving child diet quality through increasing fruit and vegetable intake, it is clear that ways to moderate children's intake of discretionary choices is an understudied area, requiring further research investment ${ }^{(20)}$. For example, a recent systematic review of interventions promoting fruit and vegetables in schools identified twenty-seven interventions ${ }^{(55)}$, compared with the four interventions targeting discretionary choices included in the current review. The mapping process undertaken in the present review also highlighted the gap in economic-type influences (such as discretionary choice cost-related initiatives, e.g. government sales tax on discretionary choices) across all settings, with no studies in this area to date. Economic-type influences consequently require consideration in further research, in particular due to the potential for economic strategies at a population level to result in a wide reduction in intakes of discretionary choices. An example in the government setting is a tax on discretionary choices, with revenue raised from the tax redirected into health initiatives or subsidising healthy foods ${ }^{(56)}$. Within the home setting economic strategies could be to highlight the cost of packaged or takeaway foods, which could reduce the approximately $30 \%$ of the weekly home food budget spent on takeaway-type foods (e.g. restaurants, clubs, fast food, takeaway) ${ }^{(57)}$

The frequency of meals away from home, a communitybased socio-cultural influence, was significantly associated with higher children's intake of discretionary choices ( $60 \%$ of studies) ${ }^{(26,27,29,41)}$. This highlights an opportunity for interventions to evaluate strategies to support parents at times when meals are consumed away from home; for example, healthy options guide or skills-based resources of nutritious foods to take when likely to consume foods away from home. Perhaps this contrasts with the higher time investment of planning home food availability by the food purchasing process that may mediate consumption, making it easier to limit discretionary choices in the home. Progress in this area will help to support parents to reduce their child's intake of discretionary choices by providing strategies to choose healthy options when consuming meals away from home, and would further be enhanced by increasing the availability of healthy meal options.
Within the home environment, when observing child feeding practices, there was a trend towards higher parental control linked with higher children's intake of discretionary choices; however, this was significant in only four out of nine studies $^{(28,30,42,47)}$. The numerous definitions and measures used to describe this feeding practice may have weakened this association and requires further investigation using standardised measures. Parental monitoring (i.e. monitoring of children's dietary intake), on the other hand, was significantly associated with higher children's intake of discretionary choices in a majority of studies measuring this exposure $^{(2,27,28,30,47,52)}$. These child feeding approaches are in line with those first described by Satter ${ }^{(58)}$, where the parent's role is to provide a range of healthy choices and the child's role is to decide which and how much of these foods he/she will consume. These findings again highlight an important role parents play in this setting. It would be of interest to further explore changes in these practices in future interventions.

Higher children's intake of discretionary choices was also associated with more frequent television use $\mathrm{e}^{(27,31,32,34)}$. This association could be influenced by food advertising or through the habits associated with screen-time activities (e.g. snacking) or a combination ${ }^{(21)}$. Although there has been extensive advocacy work to reduce food advertising during children's television viewing times ${ }^{(59,60)}$, change in home policies regarding television use is another possible solution. Based on the findings of the included studies, a change in parents' television viewing rules (policies) is likely to reduce children's intake of discretionary choices, as well as to support national guidelines regarding such sedentary activities $^{(61)}$

Observational and intervention studies in the government setting are lacking. Although there have only been three interventions, these are of particular interest given the ability for enablers at this level to have a greater influence and reach to all groups within the community (e.g. difficult-toengage and vulnerable populations) ${ }^{(15)}$. Government strategies would also support other settings by facilitating healthy options to become easy choices for consumers. Two of the three interventions to date evaluated kilojoule labelling of fast-food menus, one finding a significant reduction in kilojoules ordered ${ }^{(36,37)}$. However, a key consideration is the use of a simulated environment, which brings to question if these findings would remain in a practice setting (i.e. when child requests for discretionary choices may influence purchasing).

Intervention strategies in the education setting, such as information workshops (including consequences of discretionary choices intake) targeting either parent or teacher attitudes and beliefs, led to significantly reduced children's intake or exposure to discretionary choices ${ }^{(39,46)}$. This sociocultural influence may therefore impact on discretionary choices provision outside the area of child feeding practices (not included in these interventions). Of note, both interventions targeted attitudes and beliefs in combination 
with other strategies (e.g. implementation of a nutrition policy). This warrants further investigation to see if these findings are replicated when studied alone or if they are only a complementary strategy.

Our ecological mapping found most studies to focus on the home environment, specifically assessing the impact of policy (e.g. family rules) or physical influences (e.g. television access) on the provision of discretionary choices. The vast majority of these studies are observational in nature and although such observational studies have been published since the $1970 \mathrm{~s}^{(40)}$, to date very few interventional studies evaluating strategies have been performed ${ }^{(31,40,44,45)}$. Further interventional research is therefore required to provide evidence to support parents who are primarily responsible for the provision of discretionary choices in this setting. For example, numerous observational studies have found that children who watch more television are provided with more discretionary choices ${ }^{(25-27,31-33,42)}$, yet we are lacking the evidence from interventions to implement strategies in public health nutrition initiatives in this area.

It is important to note that discretionary choices encompass more than single groupings, such as sugarsweetened beverages or fast foods. From the present review it appears targeting the whole discretionary choices group in interventions may be perceived as too challenging, with only three (out of ten) interventions addressing a larger portion of this group. Comparatively, approximately three-quarters of observational studies included numerous discretionary choices. This is a key example where intervention design could be strengthened, as strategies would be more likely to produce the same findings when implemented in practice when controlling for changes within the discretionary choices group.

A key review strength was utilising the ecological approach with a broad search and outcome inclusion to examine the scope of current literature and identify implications for further research. Reviewer error/bias was minimised by the use of two reviewers when possible. When not, a consultative approach with two experienced reviewers was used. The quality of the review was also strengthened by the use of a validated critical appraisal tool and the PRISMA (Preferred Reporting Items for Systematic Reviews and Meta-Analyses) checklist for systematic review reporting ${ }^{(23,62)}$.

The present review is not without limitations. First, only a small body of lower-quality evidence was available. The variety of outcome measures, discretionary choices targeted and range of exposures and strategies explored did lend the review to a heterogeneous sample. It was therefore not possible to conduct a meta-analysis. It did, however, support the ecological mapping and guidance for future research. The sample heterogeneity and limited detail reported in original studies also restricted the ability to report collective magnitudes of intervention effects. For this reason, only qualitative comparisons were included.

Reporting bias within data collection is a risk, even with the aid of validated and reliable tools. The discretionary choices focus minimised the effect of under-reporting bias on findings presented. Reporting bias was also reduced in several interventions using objective experimenter observations where blinding was not possible. As the review was limited to published information, due to limited resources, publication bias may have affected the quality of assessment with several quality areas rated unknown. Although outside the scope of the current review, it is possible there is a body of practice-based evidence that would add to the findings presented.

In order to enhance the literature in the area, and to take steps towards reducing children's intake of discretionary choices, several recommendations have been formulated. More high-quality research is required across the ecological framework, but in particular in the gaps identified as noted earlier. In addition, studies that focus on multiple levels of influence would provide valuable insight to interrelated factors within different settings and environment types. Modelling these multiple influences in interventions will also assist in the application of these findings to practice. To support strengthening the quality of future studies, researchers should report findings in line with the STROBE (Strengthening the Reporting of Observational Studies in Epidemiology) or CONSORT (Consolidated Standards of Reporting Trials) statements, for the relevant study design ${ }^{(63,64)}$. To give confidence in findings, prospective longitudinal research designs with appropriate adjustment for confounders (e.g. parental education, socio-economic status, family structure, weight status) should be utilised.

\section{Conclusion}

The ecological mapping of the literature in the present review has provided a clear, practical view of the evidence to date relevant to public health nutrition. Although far more research is needed before seeing an influence in public health nutrition practice, the review highlights a step in the right direction, with studies now starting to focus on discretionary choices. The review makes a key contribution to public health nutrition research by identifying the gaps in the literature to ensure future studies address the areas of greatest unmet need. Additionally, the review findings will guide the focus of future interventions to develop multitarget initiatives to make healthy options the easy choices (i.e. through government and economic-type influences) and create a change in culture in order to reduce provision of discretionary choices. It is essential that research continues to explore predictors and potential strategies in this young age group, in order to intervene early and start making meaningful changes to improve diet quality.

\section{Acknowledgements}

Financial support: This research received no specific grant from any funding agency in the public, commercial or 
not-for-profit sectors. R.K.G. is supported by a National Heart Foundation Fellowship. Conflict of interest: None. Authorship: All authors developed the review protocol; B.J.J. conducted the literature review and drafted the manuscript; R.K.G. and G.A.H. were involved in screening and reviewing the literature, and provided guidance throughout. All authors reviewed, edited and approved the final manuscript. Ethics of buman subject participation: Ethics approval was not required for this review.

\section{Supplementary material}

To view supplementary material for this article, please visit http://dx.doi.org/10.1017/S1368980015002992

\section{References}

1. Aktas Arnas Y (2006) The effects of television food advertisement on children's food purchasing requests. Pediatr Int 48, 138-145.

2. Murashima M, Hoerr SL, Hughes SO et al. (2012) Feeding behaviors of low-income mothers: directive control relates to a lower BMI in children, and a nondirective control relates to a healthier diet in preschoolers. Am J Clin Nutr $\mathbf{9 5}$, 1031-1037.

3. Musher-Eizenman DR, Young KM, Laurene K et al. (2010) Children's sensitivity to external food cues: how distance to serving bowl influences children's consumption. Health Educ Behav 37, 186-192.

4. US Department of Health and Human Services \& US Department of Agriculture (2005) Dietary Guidelines for Americans 2005, 6th ed. Washington, DC: US Government Printing Office.

5. National Health and Medical Research Council (2013) Australian Dietary Guidelines. Canberra: NHMRC.

6. Wang Y \& Lobstein T (2006) Worldwide trends in childhood overweight and obesity. Int J Pediatr Obes 1, 11-25.

7. Australian Bureau of Statistics (2013) 4364.0.55.003 - Australian Health Survey: Updated Results, 2011-2012. http://www.abs. gov.au/AUSSTATS/abs@.nsf/Lookup/4364.0.55.003main+ features12011-2012 (accessed May 2014).

8. National Health and Medical Research Council (2013) Educator Guide. Canberra: NHMRC.

9. National Health and Medical Research Council (2011) $A$ Modelling System to Inform the Revision of the Australian Guide to Healthy Eating. Canberra: NHMRC.

10. Krebs-Smith SM, Guenther PM, Subar AF et al. (2010) Americans do not meet federal dietary recommendations. J Nutr 140, 1832-1838.

11. Commonwealth Scientific Industrial Research Organisation, Preventative Health National Research Flagship \& University of South Australia (2008) 2007 Australian National Children's Nutrition and Physical Activity Survey - Main Findings. Canberra: Department of Health and Ageing, Australian Food and Grocery Council, Department of Agriculture Fisheries and Forestry.

12. Australian Bureau of Statistics (2014) 4364.0.55 - Australian Health Survey: Nutrition First Results - Foods and Nutrients, 2011-12. http://www.abs.gov.au/ausstats/abs@.nsf/Lookup/ 4364.0.55.007main+features12011-12 (accessed January 2015).

13. Department of Health \& Food Standards Agency (2012) National Diet and Nutrition Survey. Headline Results from Years 1, 2 and 3 (Combined) of the Rolling Programme (2008) 2009-2010/11). London: Department of Health and FSA.
14. Crockett SJ \& Sims LS (1995) Environmental influences on children's eating. J Nutr Educ 27, 235-249.

15. Swinburn B, Egger G \& Raza F (1999) Dissecting obesogenic environments: the development and application of a framework for identifying and prioritizing environmental interventions for obesity. Prev Med 29, 563-570.

16. Hirst M, Jervis N, Visagie $\mathrm{K}$ et al. (2011) Transition to Primary School: A Review of the Literature. Canberra: Department of Health and Ageing.

17. Birch L \& Ventura A (2009) Preventing childhood obesity: what works? Int J Obes (Lond) 33, Suppl. 1, S74-S81.

18. Kuhl ES, Clifford LM \& Stark LJ (2012) Obesity in preschoolers: behavioural correlates and directions for treatment. Obesity (Silver Spring) 20, 3-29.

19. Waters E, de Silva-Sanigorski A, Burford BJ et al. (2011) Interventions for preventing obesity in children. Cochrane Database Syst Rev issue 12, CD001871.

20. Evans CEL, Christian MS, Cleghorn CL et al. (2012) Systematic review and meta-analysis of school-based interventions to improve daily fruit and vegetable intake in children aged 5 to 12 y. Am J Clin Nutr 96, 889-901.

21. Cairns G, Angus K, Hastings G et al. (2013) Systematic reviews of the evidence on the nature, extent and effects of food marketing to children. A retrospective summary. Appetite 62, 209-215.

22. Higgins JPT \& Green S (2008) Cochrane Handbook for Systematic Reviews of Interventions: The Cochrane Collaboration. http://onlinelibrary.wiley.com/doi/10.1002/9780470712184. fmatter/pdf (accessed February 2014).

23. Thomas B, Ciliska D, Dobbins M et al. (2004) A process for systematically reviewing the literature: providing the research evidence for public health nursing interventions. Worldviews Evid Based Nurs 1, 176-184.

24. Deeks J, Dinnes J, D'Amico R et al. (2003) Evaluating nonrandomised intervention studies. Health Technol Assess 7, 1-186.

25. Andaya AA, Arredondo EM, Alcaraz JE et al. (2011) The association between family meals, TV viewing during meals, and fruit, vegetables, soda, and chips intake among Latino children. J Nutr Educ Behav 43, 308-315.

26. Erinosho TO, Dixon LB, Young C et al. (2013) Caregiver food behaviours are associated with dietary intakes of children outside the child-care setting. Public Health Nutr 16, 1263-1272.

27. Lopez NV, Ayala GX, Corder K et al. (2012) Parent support and parent-mediated behaviors are associated with children's sugary beverage consumption. J Acad Nutr Diet 112, 541-547.

28. Aguirre T, Hudson DB, Weber K et al. (2012) Mexican American mothers' eating and child feeding behaviors. Issues Compr Pediatr Nurs 35, 4-23.

29. Ayala GX, Rogers M, Arredondo EM et al. (2008) Away-from-home food intake and risk for obesity: examining the influence of context. Obesity (Silver Spring) 16, 1002-1008.

30. Arredondo EM, Elder JP, Ayala GX et al. (2006) Is parenting style related to children's healthy eating and physical activity in Latino families? Health Educ Res 21, 862-871.

31. Galst JP \& White MA (1976) The unhealthy persuader: the reinforcing value of television and children's purchase influence attempts at the supermarket. Child Dev 47, 1089-1096.

32. Isler L, Popper H \& Ward S (1987) Children's purchase requests and parental responses: results from a diary study. J Advert Res 27, 28-39.

33. Tabak RG (2011) Home environment and child diet. Dissert Abstr Int Sect B Sci Eng 71, 7-B.

34. Taras HL, Sallis JF, Patterson TL et al. (1989) Television's influence in children's diet and physical activity. J Dev Behav Pediatr 10, 176-180.

35. Wroten KC, O'Neil CE, Stuff JE et al. (2012) Resemblance of dietary intakes of snacks, sweets, fruit, and vegetables 
among mother-child dyads from low income families. Appetite 59, 316-323.

36. Tandon PS, Wright J, Zhou C et al. (2010) Nutrition menu labeling may lead to lower-calorie restaurant meal choices for children. Pediatrics 125, 244-248.

37. Stutts MA, Zank GM, Smith KH et al. (2011) Nutrition information and children's fast food menu choices. J Consum Aff 45, 52-86.

38. Birch LL, Johnson SL, Jones MB et al. (1993) Effects of a nonenergy fat substitute on children's energy and macronutrient intake. Am J Clin Nutr 58, 326-333.

39. Arcan C, Hannan PJ, Himes JH et al. (2013) Intervention effects on kindergarten and first-grade teachers' classroom food practices and food-related beliefs in American Indian reservation schools. J Acad Nutr Diet 113, 1076-1083.

40. Dickin KL, Hill TF \& Dollahite JS (2014) Practice-based evidence of effectiveness in an integrated nutrition and parenting education intervention for low-income parents. J Acad Nutr Diet 114, 945-950.

41. Spurrier NJ, Magarey A, Golley R et al. (2008) Relationships between the home environment and physical activity and dietary patterns of preschool children: a crosssectional study. Int J Behav Nutr Phys Act 5, 31.

42. Campbell K, Crawford D \& Ball K (2006) Family food environment and dietary behaviors likely to promote fatness in 5-6 year-old children. Int J Obes (Lond) 30, 1272-1280.

43. Campbell K, Hesketh K, Silverii A et al. (2010) Maternal self-efficacy regarding children's eating and sedentary behaviours in the early years: associations with children's food intake and sedentary behaviours. Int J Pediatr Obes 5, 501-508

44. Duncanson K, Burrows T \& Collins C (2013) Effect of a low-intensity parent-focused nutrition intervention on dietary intake of 2- to 5-year olds. J Pediatr Gastroenterol Nutr 57, 728-734.

45. Fletcher A, Wolfenden L, Wyse R et al. (2013) A randomised controlled trial and mediation analysis of the "Healthy Habits', telephone-based dietary intervention for preschool children. Int J Behav Nutr Phys Act 10, 43-54.

46. Zask A, Adams JK, Brooks LO et al. (2012) Tooty Fruity Vegie: an obesity prevention intervention evaluation in Australian preschools. Health Promot J Aust 23, 10-15.

47. Brown KA, Ogden J, Vogele C et al. (2008) The role of parental control practices in explaining children's diet and BMI. Appetite 50, 252-259.

48. Buck C, Bornhorst C, Pohlabeln H et al. (2013) Clustering of unhealthy food around German schools and its influence on dietary behavior in school children: a pilot study. Int $J$ Behav Nutr Phys Act 10, 65-75.

49. Gubbels J, Kremers S, Goldbohm R et al. (2012) Energy balance-related behavioural patterns in 5-year-old children and the longitudinal association with weight status development in early childhood. Public Health Nutr 15, 1402-1410.
50. Gubbels J, Kremers S, Stafleu A et al. (2011) Association between parenting practices and children's dietary intake, activity behavior and development of body mass index: the KOALA Birth Cohort Study. Int J Behav Nutr Phys Act 8, 18-31.

51. Kerr MA, McCrorie TA, Rennie KL et al. (2010) Snacking patterns according to location among Northern Ireland children. Int J Pediatr Obes 5, 243-249.

52. Ogden J, Reynolds R \& Smith A (2006) Expanding the concept of parental control: a role for overt and covert control in children's snacking behaviour? Appetite 47, 100-106.

53. Vereecken C, Huybrechts I, Maes L et al. (2008) Food consumption among preschoolers: does the school make a difference? Appetite 51, 723-726.

54. Bevelander KE, Anschutz DJ \& Engels R (2012) The effect of a fictitious peer on young children's choice of familiar $v$. unfamiliar low- and high-energy-dense foods. Br J Nutr 108, 1126-1133.

55. Delgado-Noguera $\mathrm{M}$, Tort $\mathrm{S}$, Martinez-Zapata MJ et al. (2011) Primary school interventions to promote fruit and vegetable consumption: a systematic review and meta-analysis. Prev Med 53, 3-9.

56. Mytton OT, Clarke D \& Rayner M (2012) Taxing unhealthy food and drinks to improve health. BMJ $\mathbf{3 4 4}$, e2931-e2938.

57. Australian Bureau of Statistics (2011) 6530.0 - Household Expenditure Survey, Australia: Detailed Expenditure Items, 2009-10. http://www.abs.gov.au/AUSSTATS/abs@.nsf/mf/ 6530.0/ (accessed January 2015)

58. Satter E (1996) Internal regulation and the evolution of normal growth as the basis for prevention of obesity in children. J Am Diet Assoc 96, 860-864.

59. Cancer Council NSW (2008) Coalition on Food Advertising to Children. http://www.cfac.net.au/index.html (accessed January 2015).

60. Al-Farsi YM, Waly MI, Al-Sharbati MM et al. (2013) Levels of heavy metals and essential minerals in hair samples of children with autism in Oman: a case-control study. Biol Trace Elem Res 151, 181-186.

61. Department of Health (2014) Australia's Physical Activity and Sedentary Behaviour Guidelines. http://www.health.gov.au/ internet/main/publishing.nsf/content/health-pubhlth-strategphys-act-guidelines (accessed January 2015).

62. Moher D, Liberati A, Tetzlaff J et al. (2009) Preferred Reporting Items for Systematic Reviews and Meta-Analyses: The PRISMA Statement. Ann Intern Med 151, 264-269.

63. Vandenbroucke J, von Elm E, Altman D et al. (2014) Strengthening the Reporting of Observational Studies in Epidemiology (STROBE): explanation and elaboration. Int J Surg 12, 1500-1524.

64. Moher D, Hopewell S, Schulz KF et al. (2010) CONSORT 2010 explanation and elaboration: updated guidelines for reporting parallel group randomised trials. BMJ 340, c869-c897. 